تأثير إضافة نشارة الخشب المعالجة على خصائص الكتل الخرسانية المصمتة والمجوفة

\author{
ياسين علي صالح القيسي \\ قسم الهندسة المدنية / كلية الهندسة / جامعة لكريت

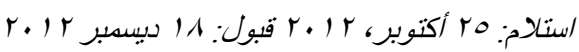

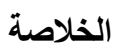

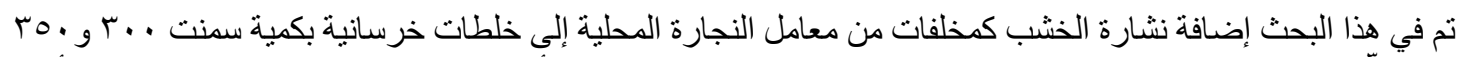

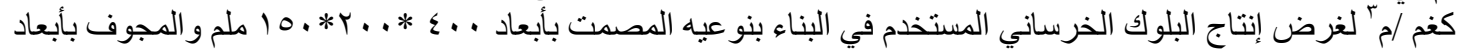

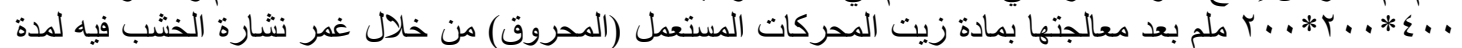

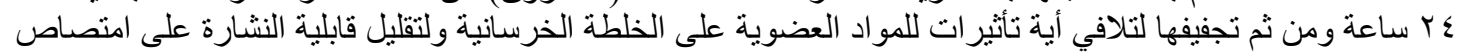

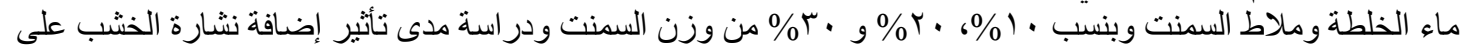

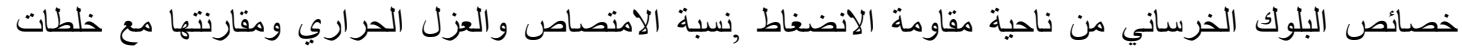

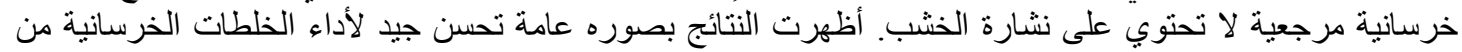

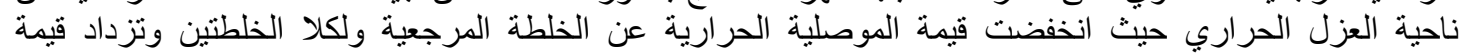

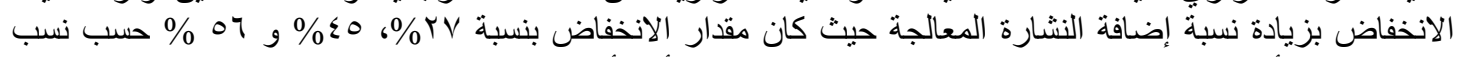

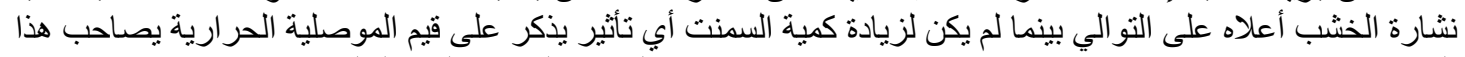

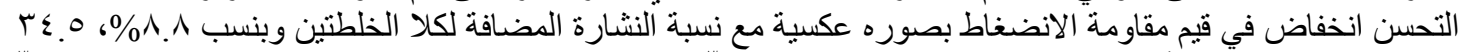

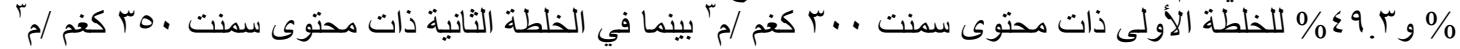

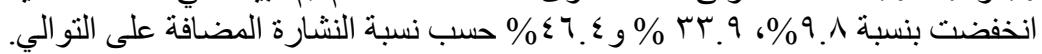

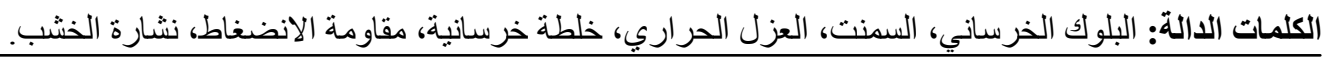

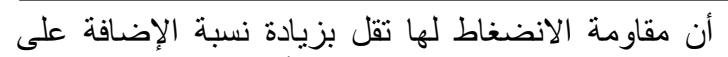

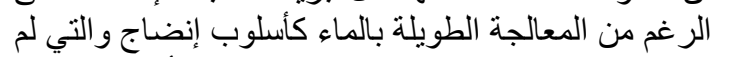
تساهم في زيادة مقاومة الانضغاط.

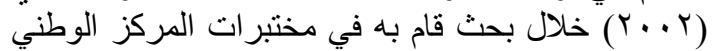

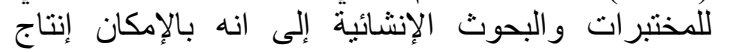

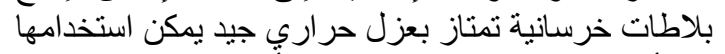

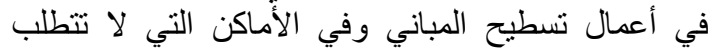

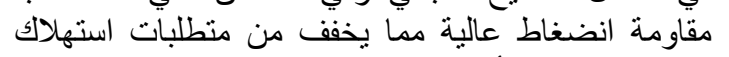

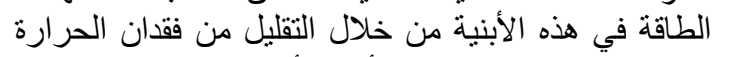

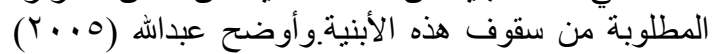

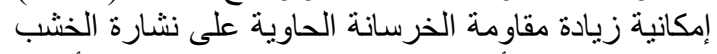

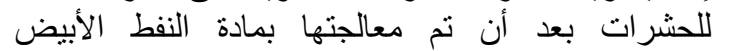

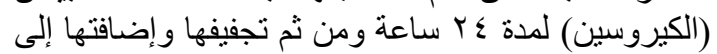

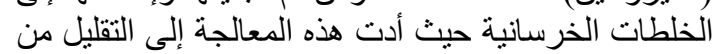

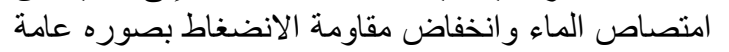

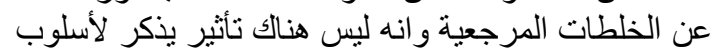

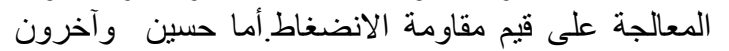

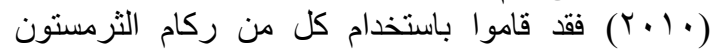
المكسر وحجر البورسيلينايت ونشارة الخشب في في في إنتاج

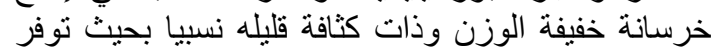

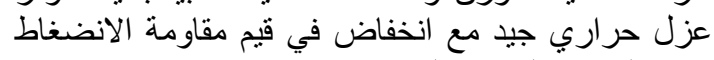

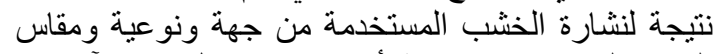
الركام المستخدم من جهة أخرى.قام توكيل دي الخدي وآخرون

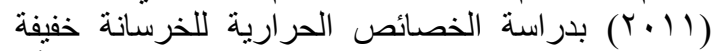

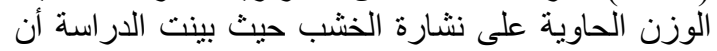

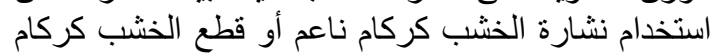

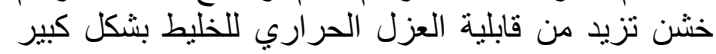

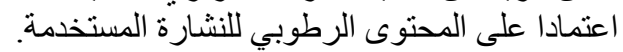

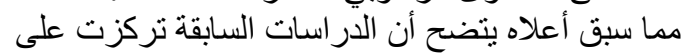

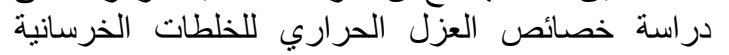

أصبحت مسالة تدوير المخلفات الصناعية وإعادة

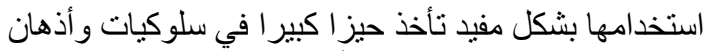

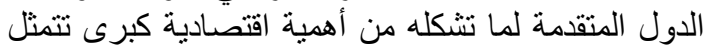

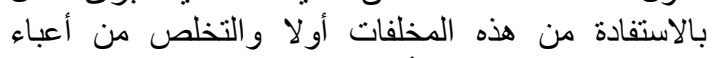

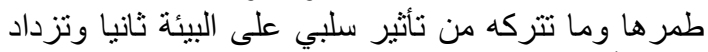

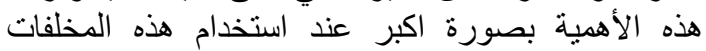

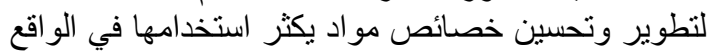

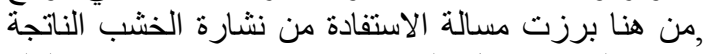

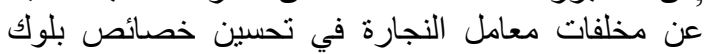

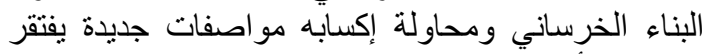

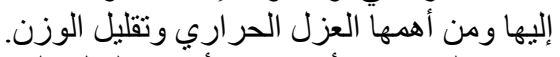

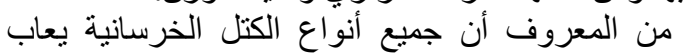

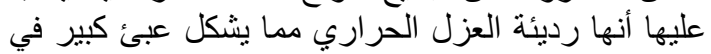

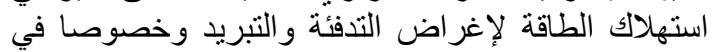
أجواء شديدة الحرارة كالتي يمر بها بلدنا لإلتلك كانت فكرة

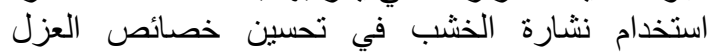

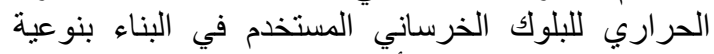

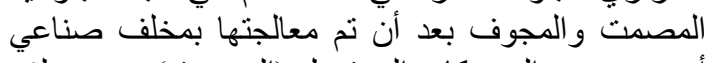

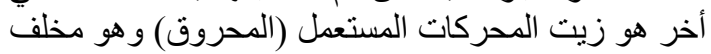

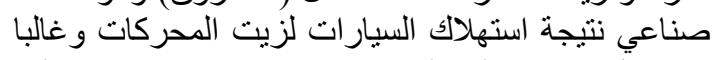

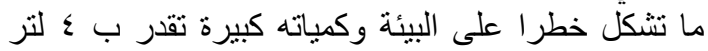

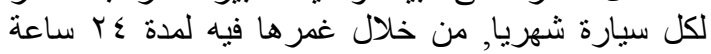

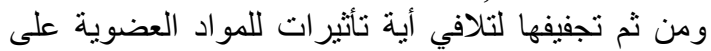
الخلطة الخرسانية.

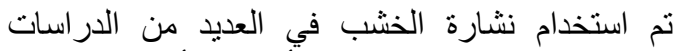
السابقة من قبل عدة باحثين حيث أُجرى الأوسي والثي النعمان

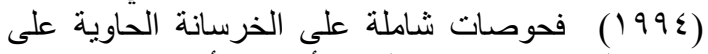

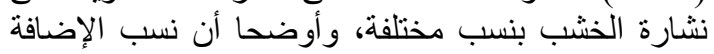

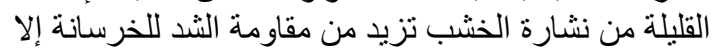




\begin{tabular}{|c|c|c|}
\hline م.ق.ع. رقم 0؛ لسنة ؛ 191 & النتائج & اسم الفحص \\
\hline لا يقل عن •Tr & $r \cdot \xi$ & النعومة (م ب/كغز) \\
\hline لا يقل عن 0؛ & Y. & وقت التماسك الابتدائي (دقيقة) \\
\hline لا يقل عن · ا ساعة & ६.TV & وقتت التماسك النهائي (ساعة) \\
\hline لا يقل عن 10 & $Y \cdot . V$ & تحمل الضغط (نت/ملم") \\
\hline لا بزيد عن ؛ & $r . \tau^{\top}$ & الفقدان عند الحرق ( \%) \\
\hline لا تزيد عن & 1.91 & المواد غير القابلة للذوبان (\%) \\
\hline لا لز تزيد عن م & $0 . \leqslant 1$ & محتوى الومينات ثلاثي أكالسيوم \\
\hline لا لاتزيد عن ^.؟ & T.Y. & محتوى ثالث اوكسيد الكبريت (\%) \\
\hline لا تزيد عن 7 & $r .97$ & محتوى اوكسيد الحديديك (\%) \\
\hline لا تزيد عن 0 & 1.19 & محتوى اوكسيد المغتسيوم (\%) \\
\hline
\end{tabular}

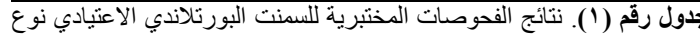

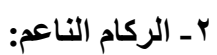

تم استخدام الركام الناعم من مقالع المدينة، الجدو لان رقم

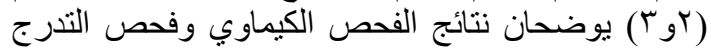

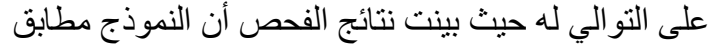

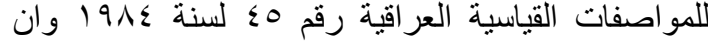

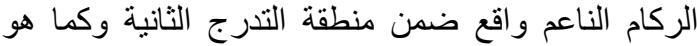

موضح في الجداول المذكورة.

\begin{tabular}{|c|c|c|}
\hline م.ق.ع. رقهم 0 ؛ 19 لسنة & 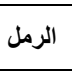 & الفحص الكيماوي \\
\hline اقل من 0. & $\because \varepsilon 1$ & نسبة المواد الجبسية \% \\
\hline اقل من r & 1.74 & نسبة الأملاح الذائبة الكلية \% \\
\hline
\end{tabular}

الحاوية على نشارة الخشب بصورة عامة دون محاولة إنة إنتاجة

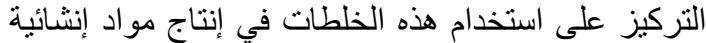
كالبلوك مثلا تستخدم في أعمال البناء كما أن البحوث الته حول معالجة نشارة الخشب لاز الت قليلة.

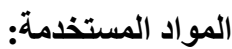

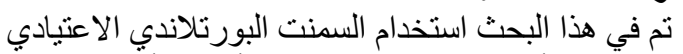

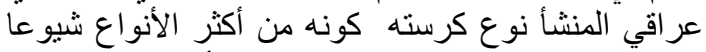

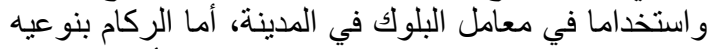

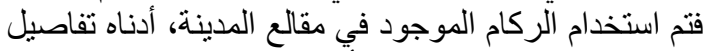

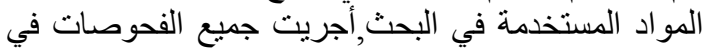
مختبر ات قسم الهندسة المدنية في جامعة تكريت.

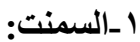

تم استخدام السهنت البورتلاندي الاعتيادي عر اقي المنشأ

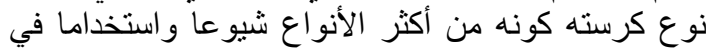

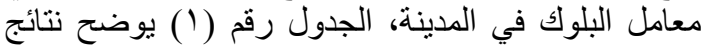
الفحوصات المختبرية وخو اصله الكيميائية و الفيزيائية و الذي لتي يبين مطابتته للمو اصفات القياسية العر اقية رقم (0؛ ؛ ) لسنة $.191 \varepsilon$

\begin{tabular}{|c|c|c|c|c|c|}
\hline \multicolumn{5}{|c|}{ م.ق.ع.رقم 0 ؛ لسنة \& 19 } & \multirow{2}{*}{ مقاس المنخل (ملم) } \\
\hline منطقة تلرج (؛) & منطقة تلرج (ץ) & منطقة تدرج (r) & منطقة تدرج (1) & الرمل \% & \\
\hline $1 \ldots$ & $1 \ldots$ & $1 \cdots$ & $1 \ldots$ & $1 \ldots$ & 9.0 \\
\hline $1 \cdots-9$. & $1 \cdots-9$. & $1 \cdots-9$. & $1 \cdots-9$. & 91.4 & $\varepsilon . V_{0}$ \\
\hline $1 \cdots .90$ & $1 \cdots-10$ & $1 \cdots-v_{0}$ & $90-7$. & $V 7.0$ & ז.r. \\
\hline $1 \cdots-9$. & $1 \cdots v_{0}$ & $9 \cdot .00$ & $V \cdot-r \cdot$ & 77.0 & 1.11 \\
\hline $1 \cdots-\Lambda$. & V9_7. & 09 - ro & $r \varepsilon_{-10}$ & $0 \Lambda$. YO $_{0}$ &. $.7 \ldots$ \\
\hline $0 .-10$ & $\leqslant 0.10$ & $r \cdot-1 \cdot$ & $T \cdot .0$ & $11 . V_{0}$ & $\cdot r \ldots$ \\
\hline 10. & $1 \cdot$. & $1 \cdot$. & $1 .$. & 0.10 &. .10 \\
\hline$\theta_{-\cdot}$ & $\theta_{-}$ & $\theta_{-\cdot}$ & $\theta_{-} \cdot$ & $\because 0$ & $\because$ Vo \\
\hline
\end{tabular}

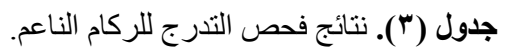

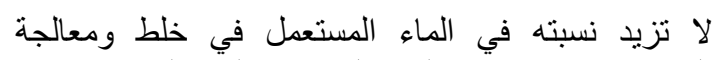

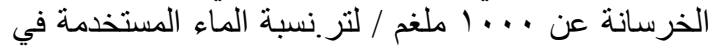

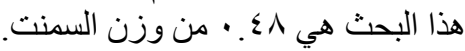

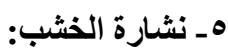

تم إجر اء التحليل المنخلي لنشارة الخشب التشب التي تم الحصول

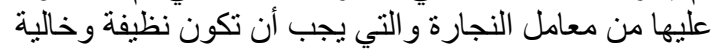

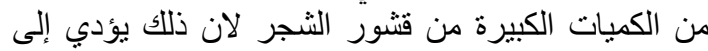

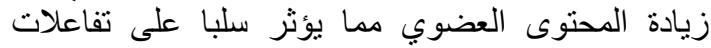

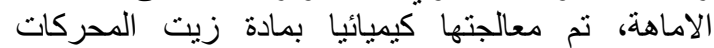

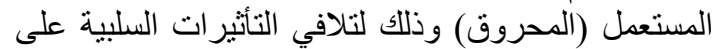

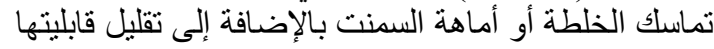

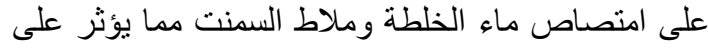

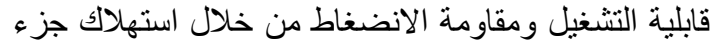

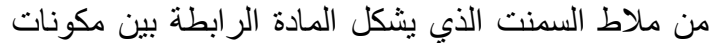
الخرسانة، الجدول (0) يوضح نتائج هذا التحليل.

\begin{tabular}{|c|c|}
\hline النسبة المئوية العابرة \% & مقاس المنخل (ملم) \\
\hline $1 \ldots$ & 9.0 \\
\hline 70.1 & $\varepsilon . V_{0}$ \\
\hline r. & T.TY \\
\hline$V .7$ & 1.11 \\
\hline
\end{tabular}

جدول (•). النسبة المئوية لنشارة الخشب المار من كل منخل
كما هو مستخدم في معامل البلوك في المدينة تم

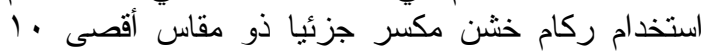

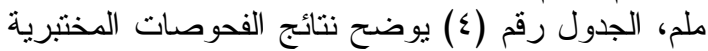
له و التي تبين انه مطابق للمو اصفات القياسية العر اقية رقم

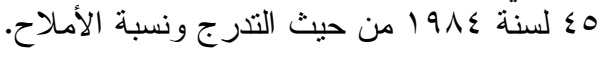

\begin{tabular}{|c|c|c|}
\hline 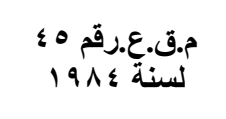 & العئوية & مقاس المنخل \\
\hline $1 \ldots-9$. & $1 \ldots$ & $1 \leqslant$ \\
\hline $10_{-} 0^{\prime}$ & $\Lambda \cdot .1$ & 1. \\
\hline $1 \cdot \ldots$ & $\wedge . \wedge$ & $\varepsilon . \vee 0$ \\
\hline لا تزيد عن 1. •\% & $\because \cdots \wedge V$ & الجبسية (المواد) \\
\hline لا تزيد عن ॰ \% & $\because .01$ & الذائبة الأملاح (\%) \\
\hline
\end{tabular}

جدول رقم (؛ ). نتائج الفحوصات المختبرية للركام الخشن. ع - الماء: تم استخدام ماء صالح للشرب في إعداد ومعالجة

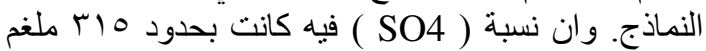

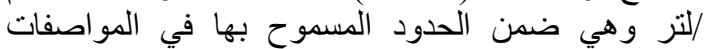

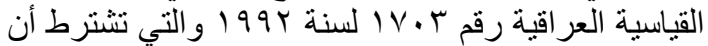




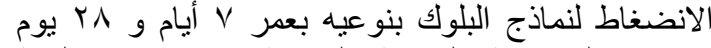

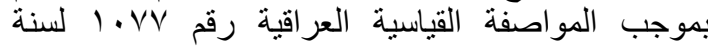
1910 بينما تم فحص الموصلية الحرارية للمكعبات بعدر

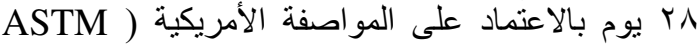

(C1058-03 and C177-10

النتائج والمناقشة:

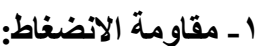
تم إجراء فحص مقاومة الإنفاطنة الإنغاط للبلوك الخرساني

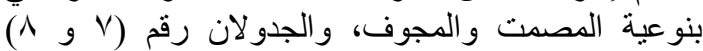

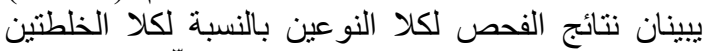

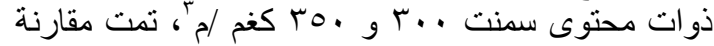

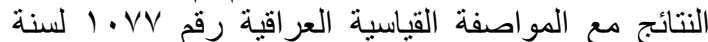

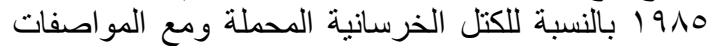

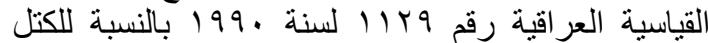
الخرسانية غير المحملة. يتضح من الجدول رقم (V) بان إضافة الفئة نشارة الخشب

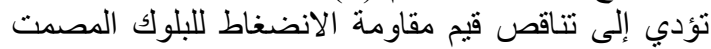

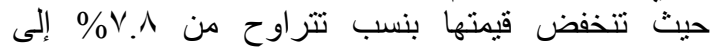

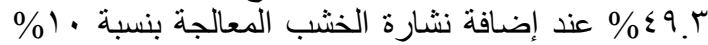

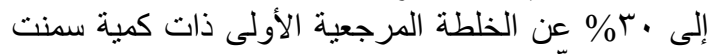

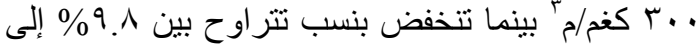

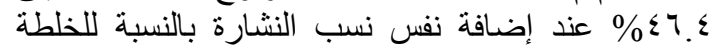

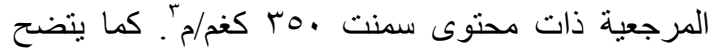

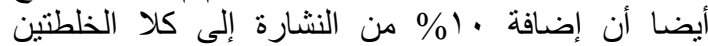

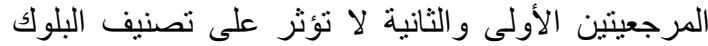

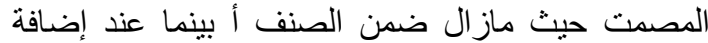
• . • الحاملة ليستقر في خانة الجدران غير الحاملة.الشكل رقم ( ) (1) يوضح هذه القيم.

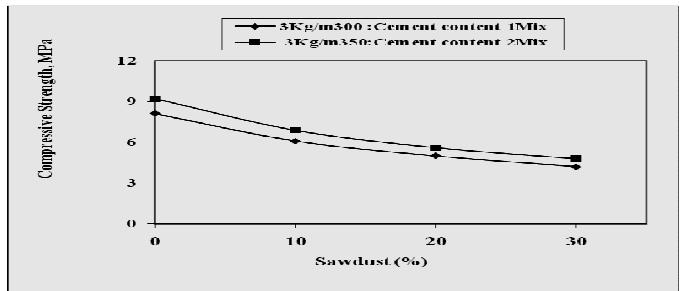

شكل رقم (1). يوضح مقاومة الانضغاط بعمر ^ بيوم للبلوك المصدت ولنسب مختلفة من نشارة الخشب النغاطي.
7 - زيت المحركات: تم استخدام زيت المحركات المستعمل (المحروق) الناتج كمخلفات صناعية لكونه لا يؤدي إلى رفع كلى كلفة الإنتاج كما

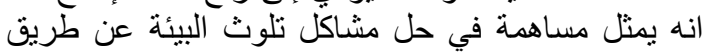

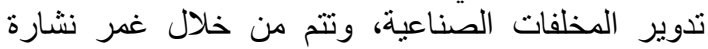

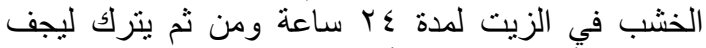

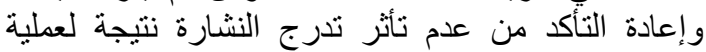

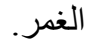

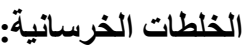

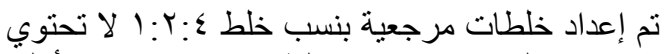

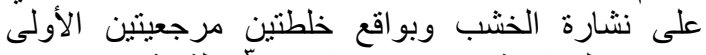

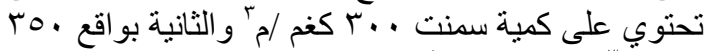

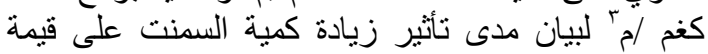

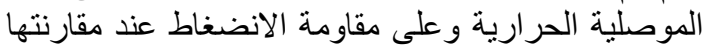
مع الخرسانة النشارية.

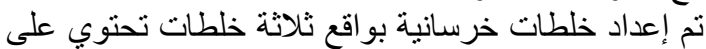

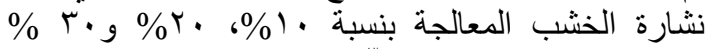

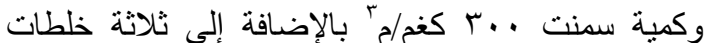

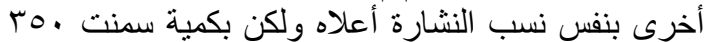

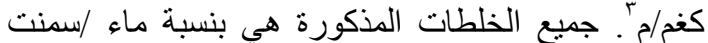

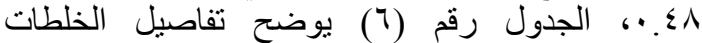
الخرسانية المستخدمة في البحث.

\begin{tabular}{|c|c|c|c|}
\hline نسبة النشارة \% & كمية السمنت /مثن & الخلطة رمز & رقمّم \\
\hline$\cdot$ & $r \ldots$ & MR1 & 1 \\
\hline 1 . & r.. & MR12 & $r$ \\
\hline$r \cdot$ & $r \ldots$ & MR13 & $r$ \\
\hline$r$. & r.. & MR14 & $\varepsilon$ \\
\hline • & ro. & MR2 & 0 \\
\hline 1 . & ro. & MR21 & 7 \\
\hline$r \cdot$ & ro. & MR22 & $\mathrm{V}$ \\
\hline$r$. & ro. & MR23 & $\Lambda$ \\
\hline
\end{tabular}

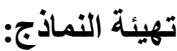

تم استخدام الخلطات الخرسانية الموضحة في الجدول

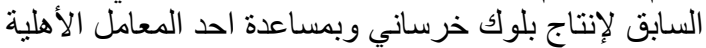

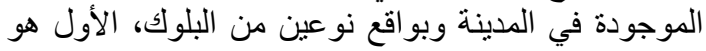

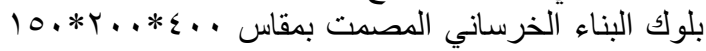

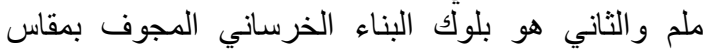

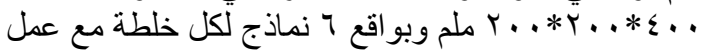

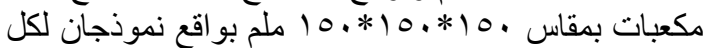

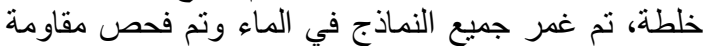

\begin{tabular}{|c|c|c|c|c|}
\hline 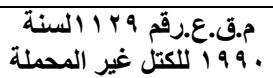 & 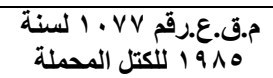 & مقاومة الانضغاط بعمر (نتملم) & 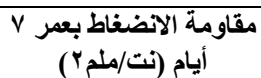 & الخلطة \\
\hline \multirow{8}{*}{ ع نت/ملم ب لمعدل r كتل } & \multirow{4}{*}{ 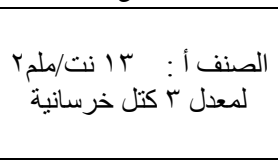 } & $1 \leqslant . Y$ & $1 \pi . r$ & MR1 \\
\hline & & 15.1 & IY.Y & MR12 \\
\hline & & 9.4 & 1.0 & MR13 \\
\hline & & $V . Y$ & $7 . \varepsilon$ & MR14 \\
\hline & \multirow{4}{*}{ لمعدل بَ كتل خرسانية 9 نتمبك } & $10 . r$ & $1 \leqslant 0$ & MR2 \\
\hline & & $1 \pi . \wedge$ & 11.9 & MR21 \\
\hline & & $1 . .1$ & 9.1 & MR22 \\
\hline & & $\Lambda . Y$ & $\Lambda . \Gamma$ & MR23 \\
\hline
\end{tabular}

جدول رقم (V). قيم مقاومة الانضغاط للبلوك المصدت. 
المرجعية ذات محتوى سمنت .0\% كغم/مبا كما بتضح

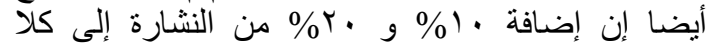

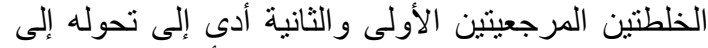

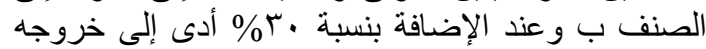

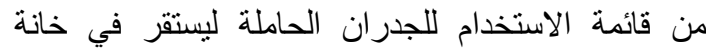
الجدران غير الحاملة.الشكل رقم (r) يمثل توضيح لهذه لانه

القيم.

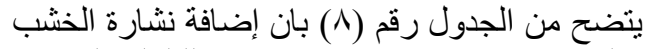

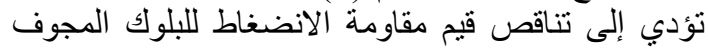

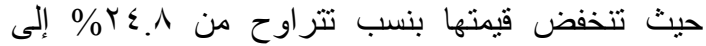

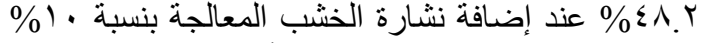

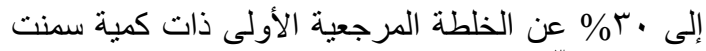

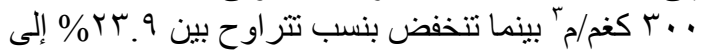

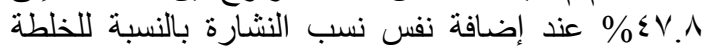

\begin{tabular}{|c|c|c|c|c|}
\hline 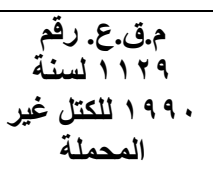 & م.ق.ع.رقم اللكتل المحملة · V لسنة & 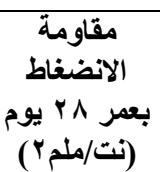 & 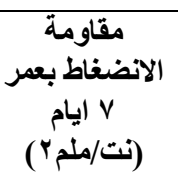 & رالخلطة \\
\hline \multirow{8}{*}{ ع نت كتل/ملمجر لمعدنية } & \multirow{4}{*}{ لمعدل r كتل خرسانية أ V نت/ملمج } & $\Lambda .1 Y$ & V. 1 & MR1 \\
\hline & & 7.1 & $7 . r$ & MR12 \\
\hline & & 0 & $\varepsilon . Y$ & MR13 \\
\hline & & $\varepsilon . Y$ & r. & MR14 \\
\hline & \multirow{4}{*}{ لمعدل بَ كتل خرسانية : هنمب } & 9.1 & ^. 1 & MR2 \\
\hline & & 7.9 & 7 & MR21 \\
\hline & & 0.7 & $\varepsilon . \wedge$ & MR22 \\
\hline & & $\varepsilon . \wedge$ & r.q & MR23 \\
\hline
\end{tabular}

جدول رقم (^). قيم مقاومة الانضغاط للبلوك المجوف.

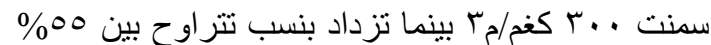

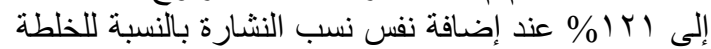

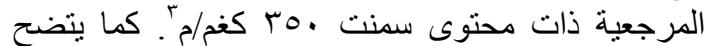

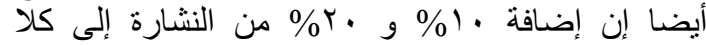

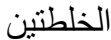

المرجعيتين الأولى والثانية تؤثران بنسبة كبيرة على الثى

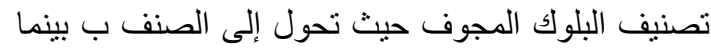

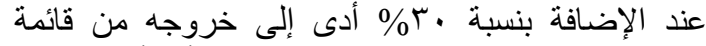

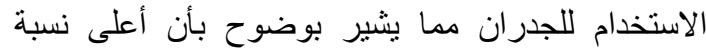

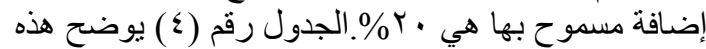

القيم.

\begin{tabular}{|c|c|c|c|}
\hline 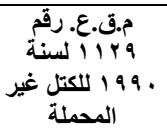 & 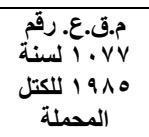 & $\begin{array}{r}\text { نامتصبةص } \\
\text { (\%) }\end{array}$ & رمز الخلطة \\
\hline \multirow{8}{*}{ 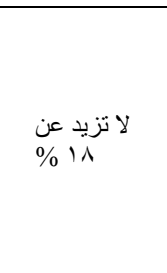 } & \multirow{4}{*}{ تزيد عن · ألا\% } & A. 1 & MR1 \\
\hline & & $1 \cdot . r$ & MR12 \\
\hline & & $1 \leq . r$ & MR13 \\
\hline & & $19 . \mathrm{V}$ & MR14 \\
\hline & \multirow{4}{*}{ تزيد عن ب: 10 لا } & $\lambda$ & MR2 \\
\hline & & $1 \cdot .1$ & MR21 \\
\hline & & $1 \leqslant .1$ & MR22 \\
\hline & & 19.0 & MR23 \\
\hline
\end{tabular}

جدول رقم (• (1). قيم نسبة الامنصاص للبلوك المجوف.

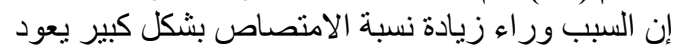

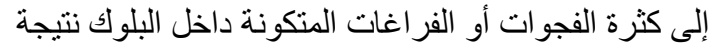

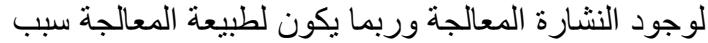

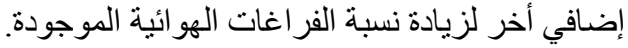
تختلف خصائص النشارة قبل وبعد المعالجة الجها فئ حيث بينت

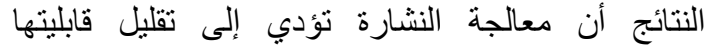

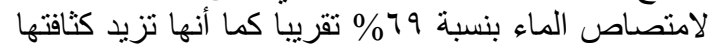

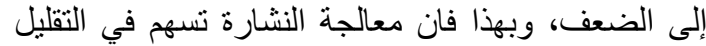

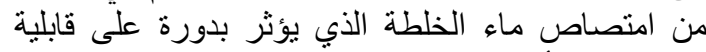

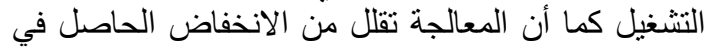

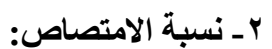

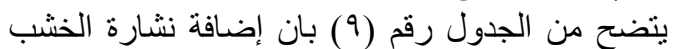

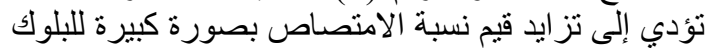

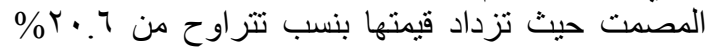

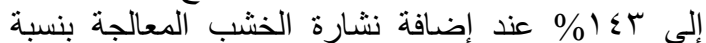

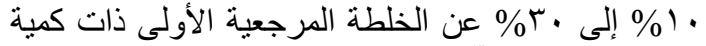

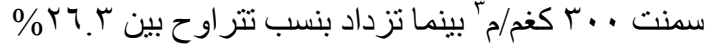

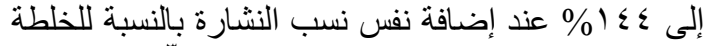

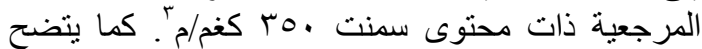

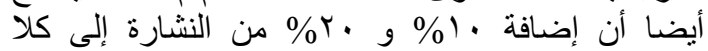
الخلطتين المرجعيتين الأولى والثانية تؤثران بنسبة كن كبيرة

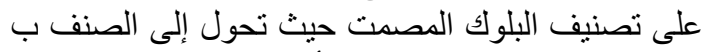

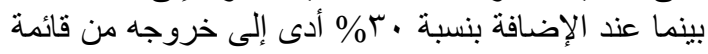

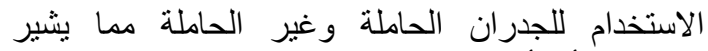

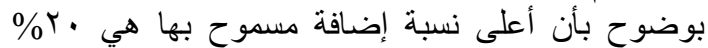
الثكل رقم (ب) يوضح هذه القيم.

\begin{tabular}{|c|c|c|}
\hline 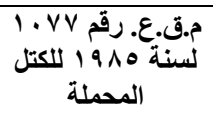 & نسبة الامتصاص & الخلطة \\
\hline \multirow{4}{*}{ الصنف أ: لاتزيد 10 \% } & $1 \cdot . r$ & MR1 \\
\hline & $10 . \mathrm{V}$ & MR12 \\
\hline & $1 \wedge . r$ & MR13 \\
\hline & TY.T & MR14 \\
\hline \multirow{4}{*}{ عن ·ب ب: لاتزيد } & $1 . .1$ & MR2 \\
\hline & 10.7 & MR21 \\
\hline & 11.1 & MR22 \\
\hline & TY.r & MR23 \\
\hline
\end{tabular}

جدول رقم (9). قيم نسبة الامتصاص للبلوك المصند.

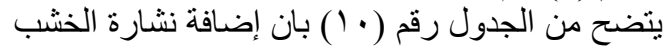

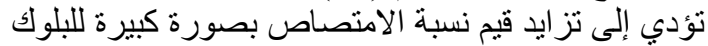

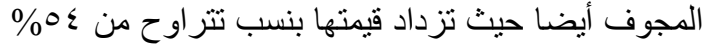

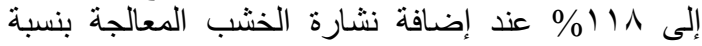

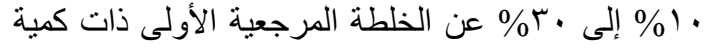


لتصل بحدود ب فولت أما التيار فيصل إلى ج. • أمبير تقريبا.

الثكل (T) يوضح قيم الموصلية الحرارية لمكعبات

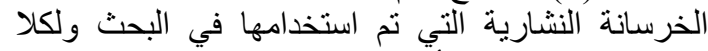

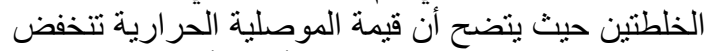

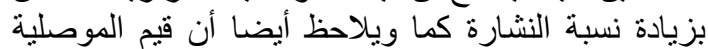

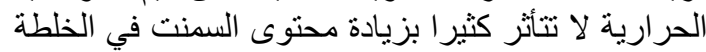

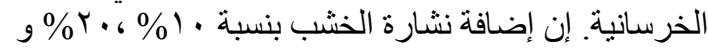

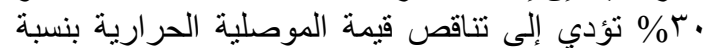

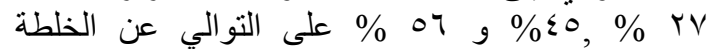

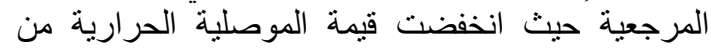

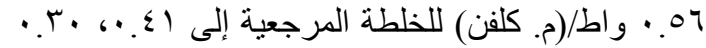

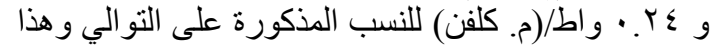

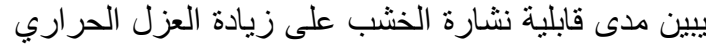

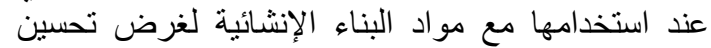

بعض خصائصها.

الاستتتاجات والتوصيات:

من خلال هذا البحث يمكن استنتاج ما يلئي:

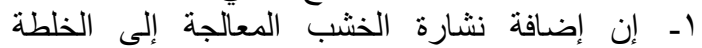

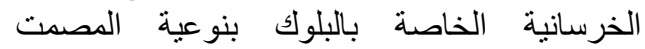

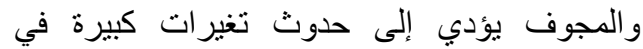

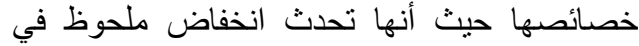

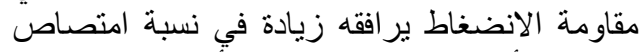

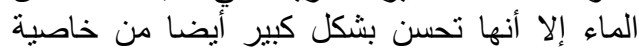

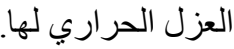

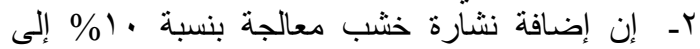

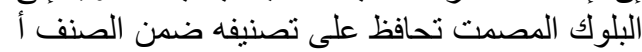

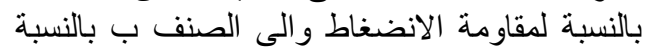

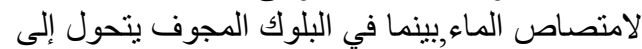

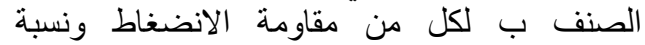

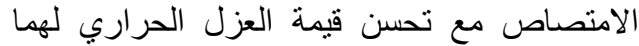
بنسبة أل Y \% \% عن الخلطة المرجعية. r- إن أعلى نسبة إضافة لنشارة الخشب بالنة بالنسبة للبلوك

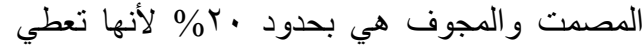

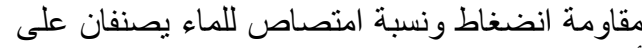

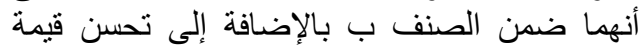

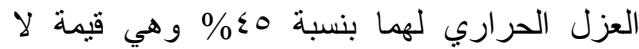
يستهان بها.

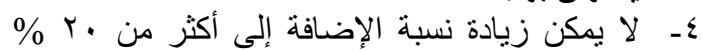

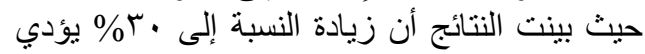

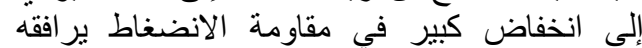

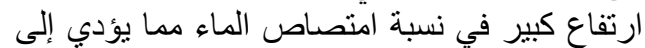

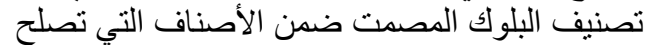

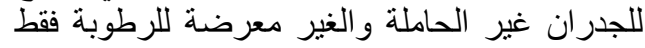

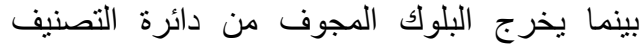
ويكون غير صالح للاستعمال.

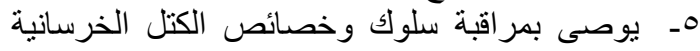

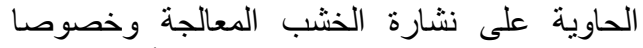

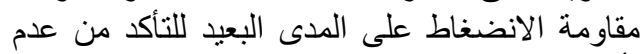

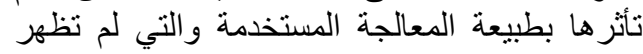

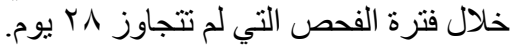

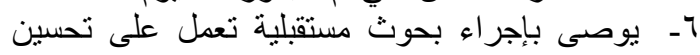
بعض الخصائص التي افتقدها البلوك الخرساني
كثافة الخلطة الخرسانية نتيجة لإضافة نشارة الخشب حيث

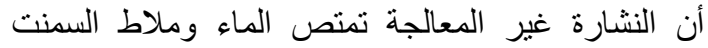

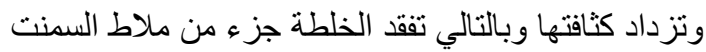

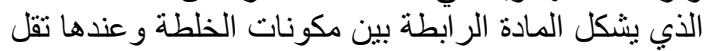

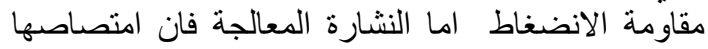
للماء وملاط السمنت اقل من النشارة غير المعالجة المالجة بكثير

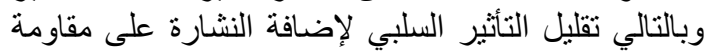

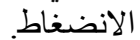

بـ الموصلية الحرارية:

احد الجوانب المهية في المرارية هذا البحث يتمثل بحساب معامل

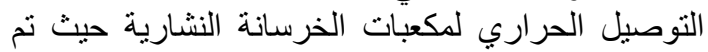

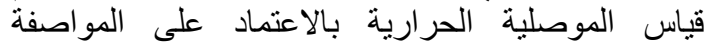
(ASTM C1058-03 and C177-10) الموصلية الحرارية تم تصنيع الجزء الأكبر منه محليا

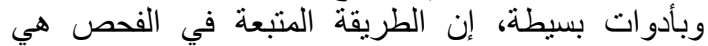

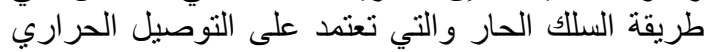

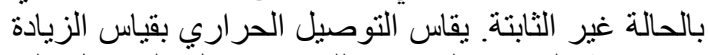

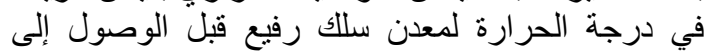

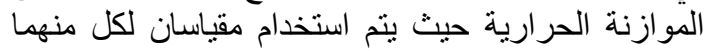

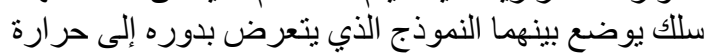
تتولد من مرور تيار كهربائي خلالله.

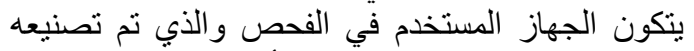

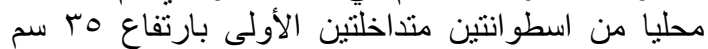

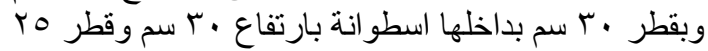

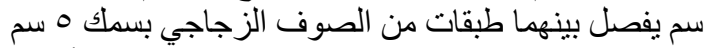
يستخدم كعازل حراري من جميع الجهات ومن الأسفات الزئل

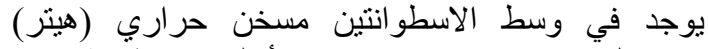
موصول بتيار كهربائي ويوجد في أعلى الاسطو انة مكان

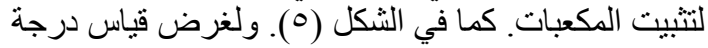

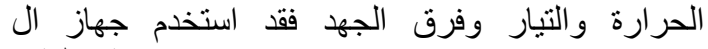
(Multimeter Digital)

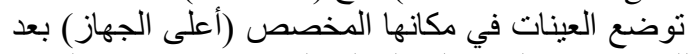
ذلك يتم ضبط القدرة الداخلة إلى المسخن عن طرين المبن التحكم

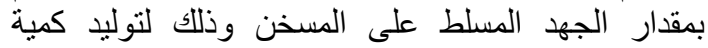

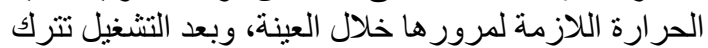

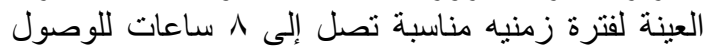

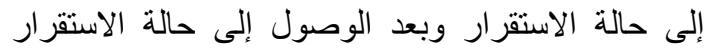

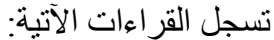
ا - الفولتيه والتيار الداخلين إلى المسخن.

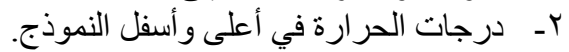

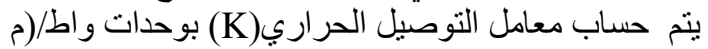
كلفن) من خلال قانون فورير وكالاتي: التوصئي التراري.

$\mathrm{P}=\mathrm{V} * \mathrm{I}=\mathrm{K} * \mathrm{~A} *(\mathrm{~T} 2-\mathrm{T} 1) / \mathrm{L}$

P V I

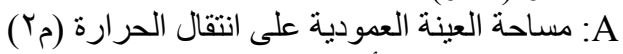

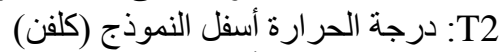

T1

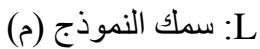

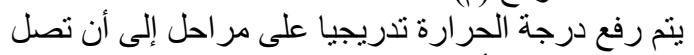

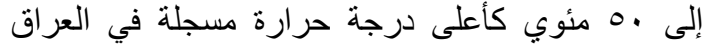
خلال فصل الصيف تقريبا ورفع الفولتيه بصورة دره تدريجية 


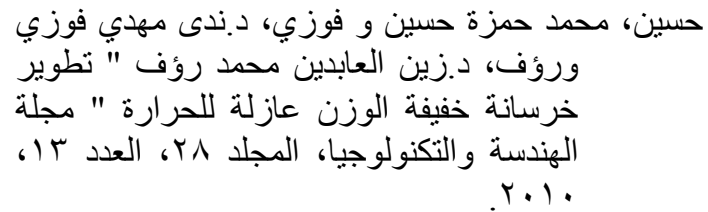

Taoukil, D., El-bouardi, A., Ezbakhe, H., and Ajzoul, T., "Thermal Properties of Concrete Lightened by Wood Aggregates", Research Journal of Applied Sciences, Engineering and Technology, Vol.3, No.2, pp. 113116, (2011).

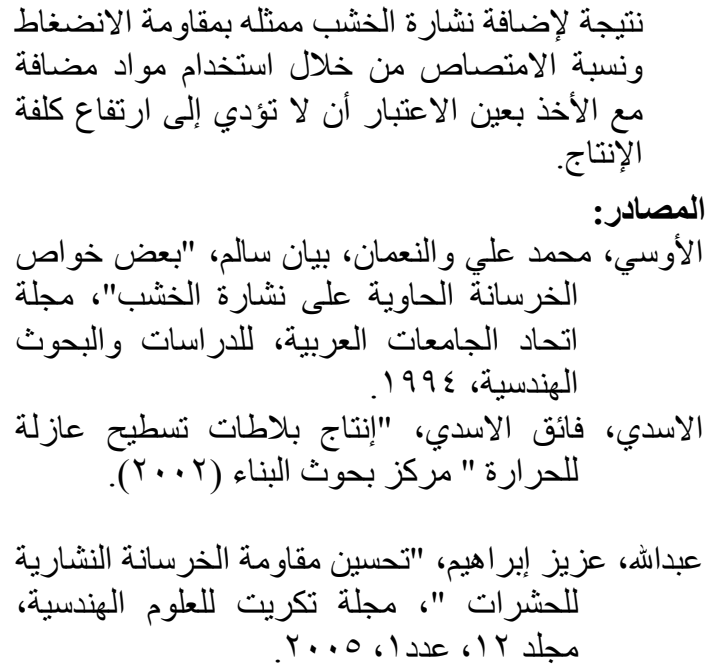

\title{
Effect of adding treated sawdust on the characteristics of the solid and hollow concrete block
}

\author{
Yaseen Ali Salih Al-Qysi
}

Civil Engineering Department / College of Engineering / University of Tikrit

\begin{abstract}
This research studies the effect of adding sawdust as waste from carpentry factory to concrete mixes quantity with cement contents 300 and $350 \mathrm{Kg} / \mathrm{m}^{3}$ to produce concrete blocks which could be used in the construction field in its both types of solid with dimensions of $400 * 200 * 150 \mathrm{~mm}$ and hollow with dimensions of $400 * 200 * 200 \mathrm{~mm}$. The sawdust material treated with oil of engines (burned) through immersed in it for 24 hours and then dried to avoid any effects of organic materials for different percentage ranged between $(10 \%$ to $30 \%)$ by weight of cement. The influence of sawdust material on the mechanical properties of concrete block, compressive strength, absorption ratio and insulation thermal has been verified. The obtained results were compared with a concrete blocks mixes with the same amount of reference cement without adding any sawdust ratio. The results indicated that using sawdust as cement replacement enhances the thermal insulation of concrete mixtures. Results showed also that the thermal conductivity decreased with increasing sawdust ratio for all concrete mixes. The decreasing ratio ranged between $27 \%$ and $56 \%$ of reference mixes, respectively. The obtained results show that the thermal conductivity doesn't effected by increasing the cement content. On the other hand, using of sawdust in concrete mix reduces the strength of concrete with ratio ranged between $8.8 \%$ to $\% 49.3$ and $9.8 \%$ to $46.4 \%$ of reference mixes, respectively.
\end{abstract}

